\title{
Monitoring Rail Infrastructure using Multisensor Navigation on a Moving Platform and Autonomous Robots
}

\author{
Simon J. Roberts, Lukasz K. Bonenberg, Hao Jing, Andrew Sowter, Xiaolin Meng, Terry Moore , Chris Hill, and Paul Bhatia, \\ University of Nottingham, $U K$
}

\section{BIOGRAPHIES}

Dr Simon Roberts work combines geospatial analysis and modelling, the development of geospatial databases, information systems design, and interactions of any system with a geospatial aspect. He is currently involved in the development dynamic mapping for predictive contextual intelligence for intelligent future transport.

Dr Lukasz Bonenberg is a Senior Experimental Officer at University of Nottingham, focusing on GNSS, low-cost navigation and sensor fusion.

Dr Hao Jing is a KTP associate at University of Nottingham, working on navigation solutions in difficult environment.

Dr Andrew Sowter is CTO of Geomatic Ventures Limited, working on the commercialisation of a novel satellite InSAR technique.

Dr Xiaolin Meng is an associate professor at the NGI, is the Course Director for MSc PNT, UK Director of Sino-UK Geospatial Engineering Centre, Faculty of Engineering.

Professor Terry Moore is the director of the NGI at the University of Nottingham; where he is the professor of Satellite Navigation. He has 30 years of research experience in surveying, positioning, and navigation technologies. He is a member of Council and a fellow of the Royal Institute of Navigation.

Dr Chris Hill is an associate professor at the NGI. He has a PhD from the University of Nottingham on the subject of Satellite Laser Ranging, and has worked on a variety of GPS and GNSS research projects over the last 20 years. He is a fellow of the RIN.

\begin{abstract}
RailSat aims to use Global Navigation Satellite System (GNSS) to monitor and maintain railway assets and its surrounding environment by railway asset owners and/or other relevant stakeholders.

The rail sector is looking for continuous monitoring solutions which have no impact on the train service, both wayside (track bound) and onboard (train bound), which require accurate positioning while travelling at high speeds (>120kmh).

This paper focuses on the combination of positioning data from traditional GNSS/INS system with processed LIDAR point cloud and discusses real-life results from the Snake Pass, Peak District, England. Data have been collected using a dedicated multisensor van but the nature of the road allows us to draw conclusions relevant to the rail industry.

This paper discusses the proposed deployment of a mobile LiDAR monitoring system consisting of a set of laser scanners and a navigation component. While the LIDAR component is capable of centimetre accuracy, it is limited by the navigation accuracy, predominantly affected by the difficult railway environment, frequent multipath and NLOS interference combined with a loss of signal next to the monitoring structures itself (bridges, cuttings, tunnels, embankments etc.), making precise positioning the biggest challenge.

The proposed navigation system combines IMU positioning system with a computer vision system capable of localisation using features in the natural environment. This paper outlines the combination of the proposed navigation system with the LIDAR's information, which provides two ways of correcting navigation trajectory in post-processing.
\end{abstract}

\section{INTRODUCTION}

The objectives of the RailSat programme is to the develop the technical capacity for the use of Global Navigation Satellite System (GNSS) in support of railway track geometry monitoring and its surrounding environment by railway asset owners and/or other relevant stakeholders. Critical assets include rail tracks, bridges, level crossings, cuttings, sidings and other infrastructure. Currently, railway infrastructure is inspected visually, by dedicated video and using measurement trains which monitor the condition of the railway network. Based on this information the track maintenance is planned and executed. However, the 
frequency of these inspections is low, track geometry, for example, is verified only once or twice a year, therefore limiting the viability and outcome of the inspections.

The rail sector is looking for continuous monitoring solutions which have no impact on the train service, both wayside (track bound) and onboard (train bound). A positioning system is therefore required to be able to accurately measure and locate track geometry and sub-decimetre track defects while travelling at high speeds $(>120 \mathrm{kmh})$. Those track defects (estimated at over 8000 at any time in the UK by Network Rail for example) would then be best serviced by the use of autonomous robots, capable of navigating to the exact location and undertaking the necessary repairs. The use of robots is essential for safety and for more efficient and timely repairs [1].

\section{IDENTIFICATION OF THE MONITORING AREAS}

Currently, railway infrastructure is inspected both visually as well as by dedicated video and measurement trains that monitor the condition of the railway network. Based on this information, track maintenance operations are planned and executed. The disadvantages of this current approach are the low frequency of both, measurement trains and visual inspections, due to the relatively high costs of increasing the frequency of these types of operations. At the same time, the UK rail network faces a pressing need to increase capacity and for service flexibility. This will not only increase the wear to the network through a higher volume of traffic but will also lead to a reduction in the time available to carry out inspection and maintenance itself [1].

The RailSAT project is aiming to address those by provides continual monitoring at a regional level through the exploitation of Sentinel-1 InSAR data to provide wide-area ground deformation rates and identify hotspots. The InSAR data can be used to provide:

- Regional analysis to qualitatively investigate overall terrain deformation trends such as slow-moving landslides, mining-induced subsidence or uplift, etc. that may have an impact on the track or corridor.

- $\quad 50-100 \mathrm{~m}$ corridor \& wide-area analysis to quantitatively investigate overall large scale terrain movement and correlation with geotechnical data (risk assessment of earthworks, landslides, mining, etc.).

Satellite monitoring offers a solution which provides asset managers and infrastructure owners with services that cover the total network, is non-intrusive, and has historical data available. InSAR data are available at a much higher frequency of every 4 to 12 days, depending upon the satellite mission employed. If this EO data source can be used as an additional indicator for monitoring track behaviour, this can supplement existing methods and overcome their weaknesses.

Interferometric Satellite Aperture Radar (InSAR) techniques make use of Synthetic Aperture Radar (SAR) datasets derived from Earth observation satellites. SAR is an active microwave imaging system and is used because it provides all-weather and cloud penetrating capabilities around the clock. Observation frequency depends on the area and extends from 4 to 12 days, historical data have a lower frequency. This service can also use data sets such as ERS and Envisat to provide baseline land deformation surveys as well as Sentinel-1 images to provide dynamic baseline surveys of wide area land deformation.

Using a novel technique call the Intermittent Small Baseline Subset (ISBAS) method [2], it is possible to make millimetric accuracy measurements of land motion, even over vegetated terrain. The technique uses stacks of satellite InSAR observations over a long period of time, deriving land motion through time-series analyses. The resulting maps of velocity have a standard error of $2 \mathrm{~mm}$ /year or better, depending on the number of observations available. The ISBAS method is operational and, when based upon freely-available Sentinel-1 data, can provide a cost effective solution to wide-area screening at low resolution.

An example ISBAS result is shown in figure 1 where more than 80 Sentinel-1 images gathered between 2015 and 2017 were processed of an area in the Netherlands. The results show severe subsidence along a bend in the railway track which may not only affect the track but also any infrastructure within a few hundred metres of the track. This result shows the average rate of motion over a 2-year period from 2015-2017.

Using this method we can not only identify cases where environmental problems affect the track but also the reverse, where continued track stress begins to affect neighbouring structures. In this case, where the track is clearly correlated to the deformation, the latter explanation is most likely.

Once those hotspots are identified, the intensive monitoring can be conducted, which includes corridor analysis to investigate track substructure deformation and stability (risk assessment of subsoil/transition points/culverts/viaducts/level crossings etc.) using a mobile LiDAR monitoring system consisting of a set of laser scanners and navigation component. While the LIDAR component is capable of centimetre accuracy, it is limited by the navigation accuracy, predominantly affected by the difficult railway environment, frequent multipath and NLOS (non-line of sight) interference combined with a loss of signal next to the monitoring structures themselves (bridges, cuttings, tunnels, embankments etc.), making precise positioning the biggest 
challenge. The following sections discuss how this situation can be improved, through the use of LIDAR data and details of monitoring itself.

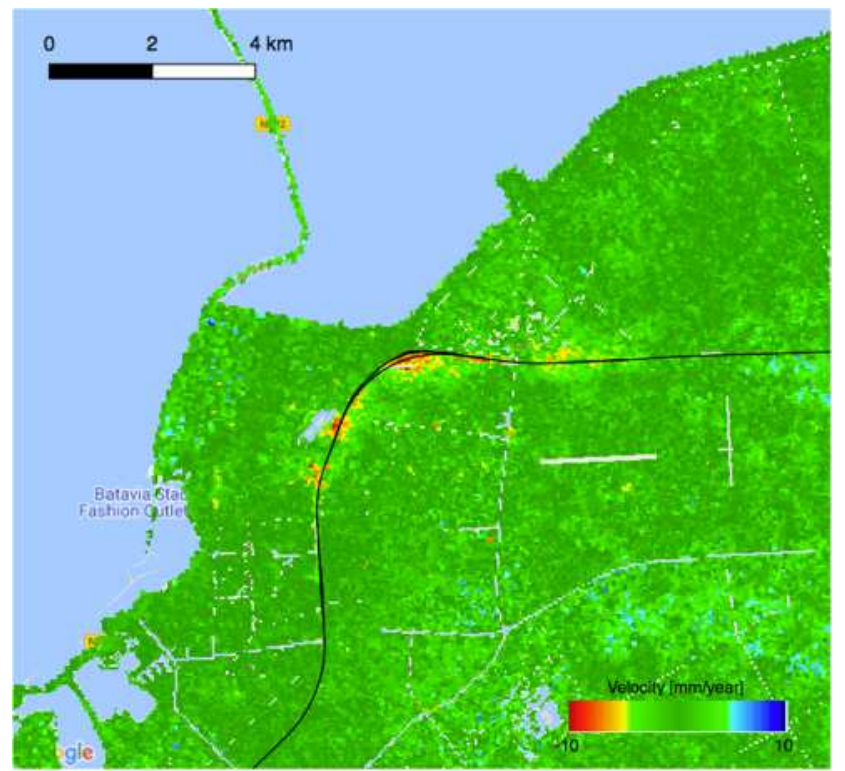

Figure 1 An ISBAS survey of an area of the close to Lelystad, Netherlands

\section{LIDAR EXPERIMENT DESCRIPTION}

To test the monitoring capabilities of 3D point clouds generated from mobile LiDAR systems, five data collections were carried out between February 2016 and July 2017. The test environment was a 10-mile-long rural single carriageway road located along the Snake Pass in the Peak District, England.

The road was selected based on several factors:

1) The area is notorious for slope failures, resulting in frequent road closures for maintenance. It was a suitable location for monitoring geo-hazards.

2) The road passes through a good mixture of environments for testing the system accuracy, including open areas and sections covered by thick foliage.

3) The environment along the road resembles the railway environment, including cuttings, embankments and poor mobile network connection [3].

Data collection was conducted using the StreetMapper mobile mapping system from 3D Laser Mapping, which can be installed on any vehicle. The system consists of a Riegl VUX-1 laser scanner, a Novatel GNSS receiver and an inertial sensor IGI IMUIIe.

The data were captured by driving both directions along the road on each survey campaing. No control points were installed during the tests, instead a combined IMU+GPS solution was used. Cuttings and foliage lead to limited sky-view and subsequent GPS gaps. The combined navigation accuracy degradation exceeding $50 \mathrm{~cm}$ in places, limiting the monitoring capacity of the system. Therefore, the post-processing of the point cloud was important to reduce the relative errors between each dataset.

The collected navigation data were post-processed in the DGNSS mode in Novatel GrafNav software, using the base station data available from UK OS-Net. GNSS data are then integrated with IMU measurements in AeroOffice, a commercial software provided by IGI. The navigation results were used to generate the 3D point cloud using 3D Laser Mapping's in-house software, MMProcess.

Table 1 Riegl VUX-1 laser scanner specification

$\begin{array}{llllll} & \text { Measurement rate } & \text { Field of view } & \text { Angle resolution } & \text { Accuracy } & \text { Precision } \\ \text { VUX-1 } & \text { Up to } 550 \mathrm{kHz} & 330 \mathrm{deg} & 0.001 \mathrm{deg} & 10 \mathrm{~mm} & 5 \mathrm{~mm} \\ \text { COMBINED POSITION } & & & & \end{array}$


The geo-hazard monitoring with the LiDAR system is based on comparing two or more point cloud datasets captured over a specific time. The detected movement is then computer, and if it exceeds a predefined threshold, the changes occurring are then flagged. However, the monitoring capability is highly dependent on the density and accuracy of the point cloud. While the density and point precision often relies on the laser scanner performance, the endpoint accuracy is highly dependent on the positioning accuracy of the navigation system[4,5], which in turn is dependent on both the hardware and the environment. As the test environment is a mixture of open sky and cuttings, the navigation result varied between centimetre level RTK position and periods where the error RMS exceeded $1 \mathrm{~m}$ (Figure 2).

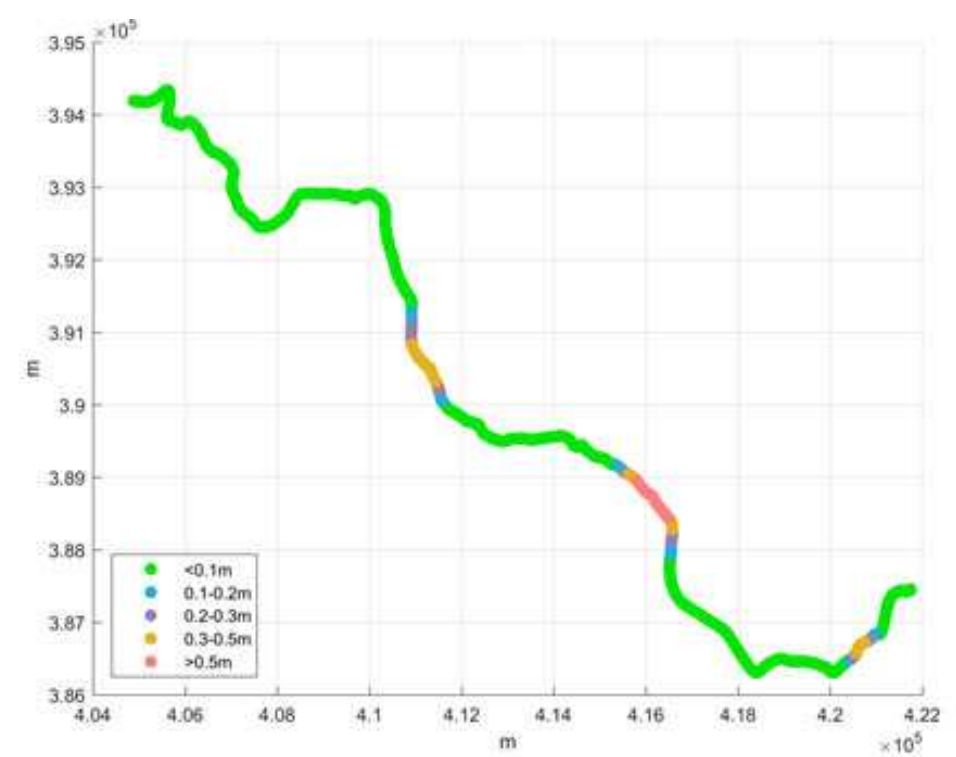

Figure 2 Easting accuracy of the combined IMU+GNSS solution

This can be improved by using point cloud data. The standard approach is to install and survey control points in areas where navigation performance is likely to degrade, e.g. going through foliage, under bridges and tunnels [3]. These points are then manually identified in the point cloud and added as the constraints during the post-processing, introducing the shift to the known position. Two main limitations of this approach are labour cost and risk to the staff, especially in the hazardous areas.

In our workflow, common features have been extracted from the point cloud and then used directly to correct the navigation information in poor coverage areas and as a result correct the matching of the point clouds. As discussed, the change detection is a de-facto comparison of two point clouds, therefore there is no need for the navigation data with absolute position. Instead, we can use features extracted from the point cloud during post-processing as "control points" to reduce a relative error between the point clouds. As shown in Figure 3 we can use this control point cloud, with the positions of the extracted features used as the reference position. We then extract the same features from the remaining datasets. Their positions are corrected towards the reference position, making all datasets consistent, with the reference point cloud, in the area of poor navigation data. This ensures that the datasets are ready for change detection and monitoring. This method eliminates the requirement of installing physical control points.

Although there is no ground truth to compare the navigation results, the accuracy is reflected in the point cloud results themselves. During our experiment, each dataset was captured by driving in both directions and thereby creating two flight-lines in the point cloud, leading to a mismatch between the point clouds captured within one dataset while driving different directions. The error between the different datasets is similarly reflected by the mismatch between the point clouds from different datasets, as discussed in the following section. 


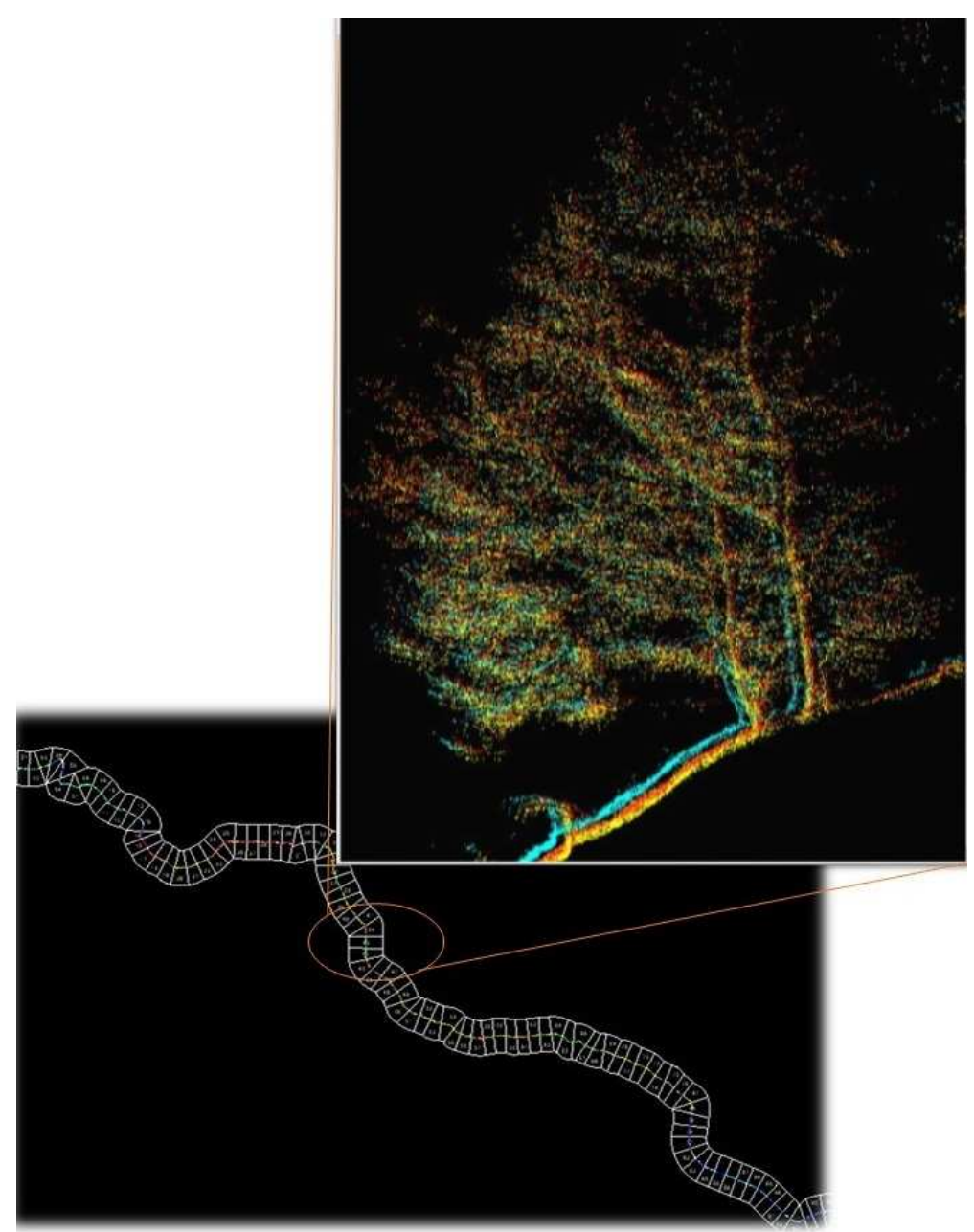

Fig 3 Example of mismatch between the point clouds

\section{ACTIVE MONITORING}

Geo-hazard monitoring with the LiDAR systems is based on comparing two or more point cloud datasets captured over a specific time. Change detection is achieved by calculating the distance between the two point clouds, that is between a point from the comparison cloud to the underlying surface represented by the reference cloud. To increase accuracy and to reduces the error caused by different point densities and the anomalies caused by scanning different feature points, the least square best fitting plane is used as the reference surface [6].

A heat map was used to display the change, with large deformations indicated as red and small values as blue. However, due to some remaining noise in the point cloud, not all detected changes are actual changes in the environment. Therefore, it is vital to differentiate between noise and detected changes. We used the following methods to reduce this noise:

a) Due to slight difference between scan position and scan angles during each data capture, the edge of the point clouds tends to show a large difference, i.e. large cloud-to-cloud distance values. In our processing, those areas are identified and not analysed.

b) The monitored area is covered with grass and other vegetation. As our data spans all seasons, from winter to the summer, many large values showing up along the roadside area are caused by the changing vegetation coverage. Those areas are also identified and removed from the analysis.

c) Misalignment caused by navigation error - those areas are corrected, following the methods discussed in the previous section, and included in the analysis.

From all the collected data, three interesting changes have been identified. In the following figures, the colour legend units are metres.

a) A damaged wall on the side of the road. This damage was not visible from a standard bird's-eye view of this section (Figure 4), although the heat-map indicated a large anomaly, which was inspected in detail and revealed a collapsed wall section requiring attention (Figure 3). 


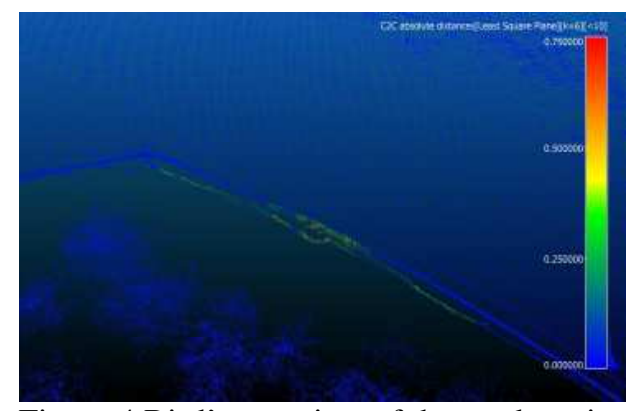

Figure 4 Bird's eye view of the road section

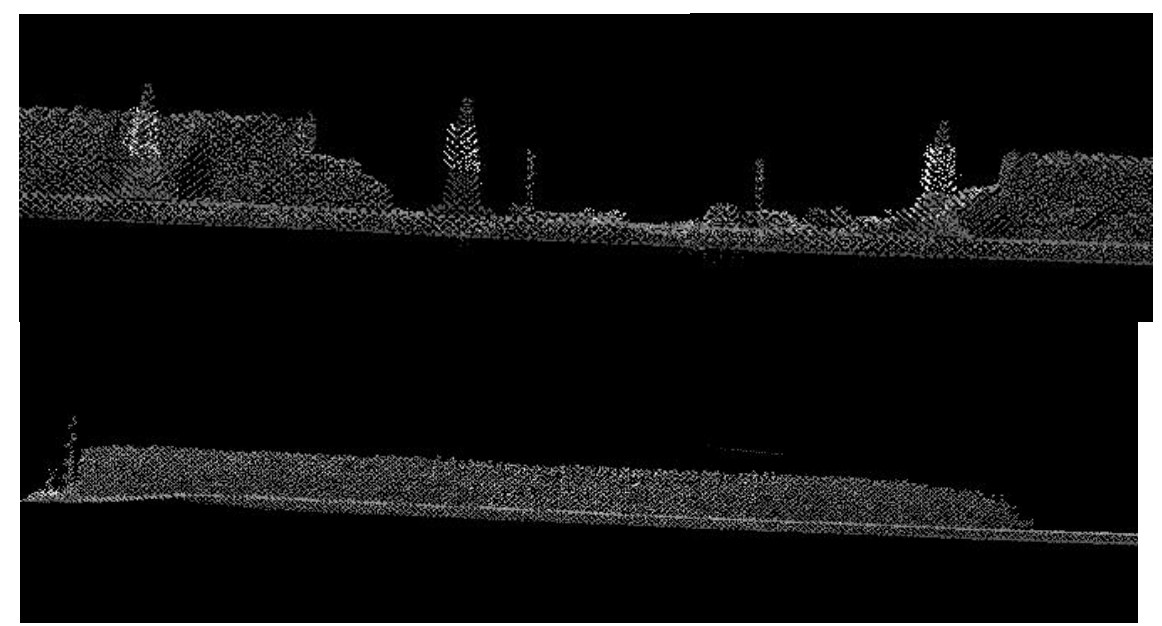

Figure 5: Wall before and after

b) Road cracking shows up very clearly in the change detection map (Figure 6). Further verification was found by inspecting point cloud (Figure 7).

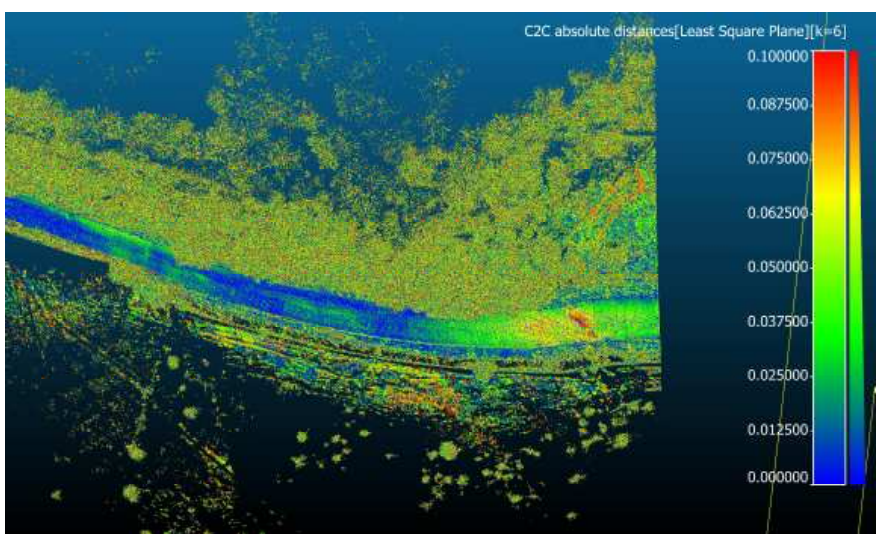

Figure 6 Change detection map showing road cracking 


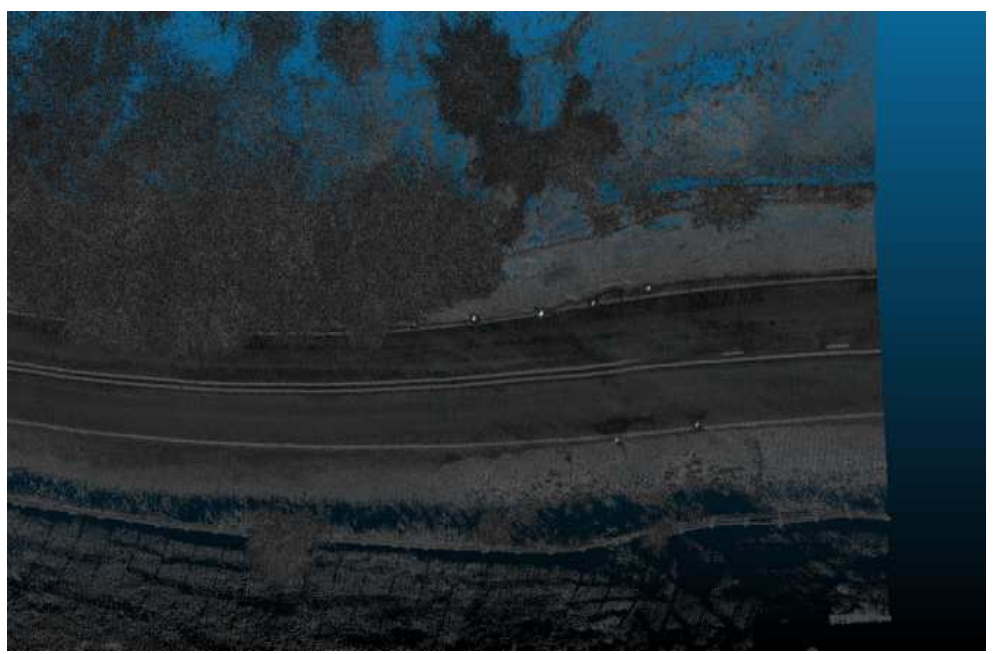

Figure 7 Detection map and point cloud

c) Continuous change found on a slope, which could be identified and validated by checking the original point cloud. These type of change are the most significant for the railway environment as early warning indicators (Figure 8).

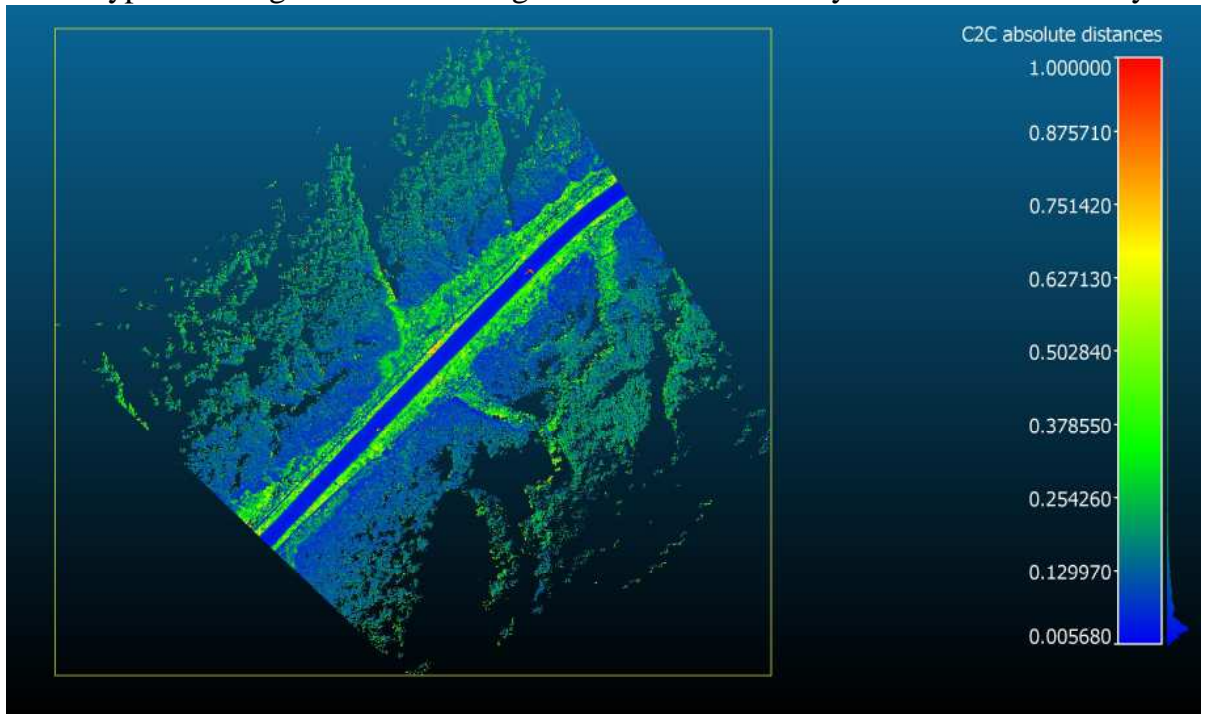

\section{SUMMARY}

This paper outlined the RailSAT continual monitoring approach using the Sentinel-1 InSAR data at the regional level, to provide wide-area ground deformation rates and identify hotspots, and LIDAR data in the hotspot area itself. The LIDAR results from the datasets captured in a relatively unstable area of the Peak District, England were used to demonstrate the feasibility of this monitoring method. This paper also demonstrated the multisensor navigation system for the rail environment. The paper focuses on the combination of positioning data from traditional GNSS/INS system combined with processed LIDAR point cloud data.

Further work will focus on developing a navigation system for the repair robots [1], using features in the natural environment in combination with low-cost GNSS/INS sensors. For the most efficient, cost-effective repairs in terms of the actual size of the repairs and disruption to the rail network, the robots need to position themselves to within $10 \mathrm{~cm}$ of the beginning and end of the fault. Such accuracy requires combined navigation system that is able first to precisely locate the damage and, in the second step, navigate the robot to the damaged section. One of the challenges here is to identify the areas of the infrastructure with poor GNSS coverage, where we have to utilise additional navigation methods. One of the suggested approaches is the use of $360^{\circ}$ images to create such a priori intelligence. A dedicated camera can be mounted on the selected trains creating a spatial database of such outages [7]. In those areas, the robot will have to use other navigation methods. As demonstrated in this paper, typical rail environment is challenging even for the combined GNSS and IMU solution. This paper discussed another positional method utilising GNSS, IMU and LIDAR to provide the combined position in such areas. 


\section{ACKNOWLEDGEMENTS}

RailSat is sponsored by the European Space Agency ARTES programme and the InnovateUK.

\section{REFERENCES}

[1] M. Taylor, M. Westwood, and A. Peters, "A modular approach to automation of condition monitoring and repair for rail," 7th IET Conference on Railway Condition Monitoring 2016 (RCM 2016), 2016.

[2] Sowter, A.; Bateson, L.; Strange, P.; Ambrose, K.; Syafiudin, M.F. DInSAR estimation of land motion using intermittent coherence with application to the South Derbyshire and Leicestershire coalfields. Remote Sens. Lett. 2013, 4(10), 979-87. DOI: 10.1080/2150704X.2013.823673

[2] Boon, D. and Evans, H., 2008. Walkover survey of a landslide on the A57 (Snake Pass) road at Cowms Moor, Derbyshire in January 2008. BGS Land Use and Development Open Report OR/08/006.

[3] Xiao, W., Vallet, B. and Paparoditis, N., 2013. Change Detection in 3D Point Clouds Acquired by a Mobile Mapping System, ISPRS Annals of Photogrammetry, Remote Sensing and Spatial Information Sciences, II-5/W2(November), pp. 331-336.

[4] Lindenbergh, R. and Pietrzyk, P., 2015. Change detection and deformation analysis using static and mobile laser scanning. Applied Geomatics 2015(7), pp. 65-74.

[5] Kaartinen, H., Hyyppa, J., Kukko, A., Jaakkola, A. and Hyyppa H., 2012. Benchmarking the Performance of Mobile Laser Scanning Systems Using a Permanent Test Field. Sensors 12(12), pp. 12814-12835.

[6] CloudCompare, 2016. CloudCompare User Manual Version 2.6.1. http://cloudcompare.org/. (March 2017).

[7] Simon J. Roberts, Lukasz K. Bonenberg, Xiaolin Meng, Terry Moore, Chris Hill, Predictive Intelligence for a Rail Traffic Management System, Proceedings of ION GNSS+ 2017 